\title{
Pressure effects on Al89La6Ni5 amorphous alloy crystallization
}

Zhuang, Yanxin; Jiang, Jianzhong; Zhou, T. J.; Rasmussen, Helge Kildahl; Gerward, Leif; Mezouar, M.; Crichton, W.; Inoue, A.

Published in:

Applied Physics Letters

Link to article, DOI:

$10.1063 / 1.1332409$

Publication date:

2000

Document Version

Publisher's PDF, also known as Version of record

Link back to DTU Orbit

Citation (APA):

Zhuang, Y., Jiang, J., Zhou, T. J., Rasmussen, H. K., Gerward, L., Mezouar, M., Crichton, W., \& Inoue, A. (2000). Pressure effects on Al89La6Ni5 amorphous alloy crystallization. Applied Physics Letters, 77(25), 41334135. https://doi.org/10.1063/1.1332409

\section{General rights}

Copyright and moral rights for the publications made accessible in the public portal are retained by the authors and/or other copyright owners and it is a condition of accessing publications that users recognise and abide by the legal requirements associated with these rights.

- Users may download and print one copy of any publication from the public portal for the purpose of private study or research.

- You may not further distribute the material or use it for any profit-making activity or commercial gain

- You may freely distribute the URL identifying the publication in the public portal 


\title{
Pressure effects on $\mathrm{Al}_{89} \mathrm{La}_{6} \mathrm{Ni}_{5}$ amorphous alloy crystallization
}

\author{
Y. X. Zhuang, J. Z. Jiang, ${ }^{\text {a) }}$ T. J. Zhou, H. Rasmussen, and L. Gerward \\ Department of Physics, Building 307, Technical University of Denmark, DK-2800 Lyngby, Denmark \\ M. Mezouar and W. Crichton \\ European Synchrotron Radiation Facility (ESRF), BP 220, 38000 Grenoble, France
}

A. Inoue

Institute of Materials Research, Tohoku University, Sendai 980-77, Japan

(Received 5 July 2000; accepted for publication 27 September 2000)

\begin{abstract}
The pressure effect on the crystallization of the $\mathrm{Al}_{89} \mathrm{La}_{6} \mathrm{Ni}_{5}$ amorphous alloy has been investigated by in situ high-pressure and high-temperature $\mathrm{x}$-ray powder diffraction using synchrotron radiation. The amorphous alloy crystallizes in two steps in the pressure range studied (0-4 GPa). The first process, corresponding to simultaneous precipitation of fcc-Al crystals and the metastable bcc$(\mathrm{AlNi})_{11} \mathrm{La}_{3}$-like phase, is governed by a eutectic reaction. The second process corresponds to the transformation of a residual amorphous alloy into fcc- $\mathrm{Al}, \mathrm{Al}_{11} \mathrm{La}_{3}, \mathrm{Al}_{3} \mathrm{Ni}$, and as yet unidentified phase(s). The applied pressure strongly affects the crystallization processes of the amorphous alloy. Both temperatures first decrease with pressure in the pressure range of $0-1 \mathrm{GPa}$ and then increase with pressure up to $4 \mathrm{GPa}$. The results are discussed with reference to competing processes between the thermodynamic potential barrier and the diffusion activation energy under pressure. (C) 2000 American Institute of Physics. [S0003-6951(00)00951-7]
\end{abstract}

Al-based amorphous alloys with an Al concentration of about 90 at. \% have attracted much attention due to their mechanical properties. ${ }^{1,2}$ The mechanical properties of partially crystallized amorphous alloys with nanometer-sized $\mathrm{Al}$ crystals could even be enhanced. ${ }^{3-5}$ These excellent mechanical properties make this new family of Al-based amorphous alloys promising candidates as advanced engineering materials. The Al-based amorphous alloys prepared are normally either thin ribbons or powders because of the high critical cooling rates required. For most engineering applications, therefore, consolidation of the amorphous ribbons or powders is applied to form bulk materials. ${ }^{4,6}$ In the consolidation process, one of the fundamental issues is the pressure effect on thermal stability of the amorphous phase. This is critical for optimizing the consolidation parameters to obtain bulk samples with the desired structure and properties. It has been demonstrated in many amorphous alloys that applied pressure affects the crystallization process. ${ }^{7-9}$ For Al-based amorphous alloys, Chen et al. ${ }^{10}$ suggested that the local atomic rearrangement instead of the long-range atomic diffusion is responsible for the formation of $\mathrm{Al}$ nanocrystals during deformation, while Ye and Lu suggested a diffusioncontrolled primary reaction for the Al precipitation. ${ }^{11}$ They also investigated the effect of pressure on the crystallization process of an $\mathrm{Al}_{89} \mathrm{La}_{6} \mathrm{Ni}_{5}$ amorphous alloy by means of piston-cylinder measurements in a pressure range of $0-1$ $\mathrm{GPa}^{8}$ Based on the analysis of the pressure-temperature curves obtained, they observed a reduction of the primary crystallization temperature $T_{x 1}$ and an enhancement of the crystallization temperature of the residual amorphous alloy $T_{x 2}$ with pressure. Volume change, instead of atomic diffu-

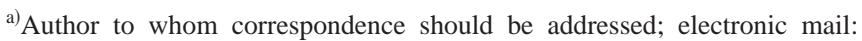
jiang@fysik.dtu.dk
}

sion, during the primary reaction was employed to explain their results. Furthermore, they predicted that Al precipitation may occur even at room temperature when applying a pressure of about $2.5 \mathrm{GPa}^{8}{ }^{8}$ In this work, we report here on in situ high-temperature and high-pressure x-ray powder diffraction (XRD) measurements using synchrotron radiation.

Ingots, with nominal composition $\mathrm{Al}_{89} \mathrm{La}_{6} \mathrm{Ni}_{5}$, were prepared by arc melting a mixture of pure $\mathrm{Al}$ ( 99.99 wt. \%), La (99.9 wt. \%), and Ni (above 99.96 wt. \%) in a purified argon atmosphere. Amorphous ribbons $\left(\sim 1.3 \times 0.02 \mathrm{~mm}^{2}\right)$ were prepared from the master alloy ingots by a single roller meltspinning apparatus. Differential scanning calorimetry (DSC) measurements were performed in a flowing purified argon atmosphere using a Perkin Elmer DSC-7 at a heating rate of $40 \mathrm{~K} / \mathrm{min}$. The amorphous alloy exhibits two exothermic crystallization reactions with the onset temperatures $T_{x 1}$ and $T_{x 2}$ of 494.1 and $611.9 \mathrm{~K}$, respectively. In situ high-pressure and high-temperature XRD measurements were performed by synchrotron radiation using the Paris-Edinburgh largevolume apparatus at the ESRF in Grenoble, France with a wavelength of $0.20215 \AA .{ }^{12}$ The sample, $2 \mathrm{~mm}$ in height, was encapsulated in a cylindrical hexagonal boron nitride container with an internal diameter of $0.8 \mathrm{~mm}$, which was used as a pressure-transmitting medium. The temperature was measured by means of a $K$-type thermocouple with a stability of $\pm 1 \mathrm{~K}$. The average heating rate in the temperature range from 298 to $673 \mathrm{~K}$ was roughly estimated to be 3 $\mathrm{K} / \mathrm{min}$. The pressure of the sample is calculated from the equation of state of $\mathrm{BN} .^{13}$

XRD patterns were recorded every $10 \mathrm{~K}$ to observe the onset temperature of crystallization within an uncertainty of $10 \mathrm{~K}$ in the pressure range of $0-4 \mathrm{GPa}$. It is found that the crystalline phases formed and the sequence of phase formation during crystallization processes of the amorphous alloy are identical in the pressure range used. Figure 1 exemplifies 


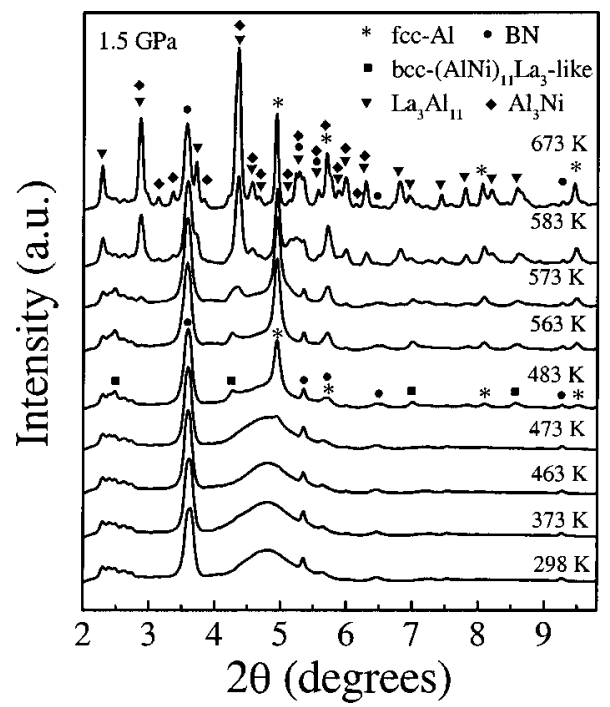

FIG. 1. In situ XRD patterns recorded at $1.5 \mathrm{GPa}$ and various temperatures for the $\mathrm{Al}_{89} \mathrm{La}_{6} \mathrm{Ni}_{5}$ amorphous alloy.

XRD patterns recorded at $1.5 \mathrm{GPa}$ and various temperatures. A broad amorphous peak, located at $2 \theta \approx 4.8^{\circ}$, together with Bragg peaks from BN marked in Fig. 1 and from the gasket in the $2 \theta$ range of $2^{\circ}-3^{\circ}$, are observed in the XRD patterns recorded at 298,373 , and $463 \mathrm{~K}$. A new tiny crystalline peak $\left(2 \theta=5^{\circ}\right)$ appears at $473 \mathrm{~K}$. At $483 \mathrm{~K}$, four new Bragg peaks located at $2 \theta \approx 2.47^{\circ}, 4.26^{\circ}, 6.98^{\circ}$, and $8.59^{\circ}$ together with Bragg peaks from fcc-Al crystals are detected. The four new Bragg peaks can be indexed to a body centered cubic (bcc) structure with a lattice constant of $6.64 \AA$ and a space group $\operatorname{Im} 3 \mathrm{~m}$. The observed and calculated interplanar spacings of the cubic phase are listed in Table I. These Bragg peaks are very close to the corresponding peaks for the orthorhombic $\mathrm{Al}_{11} \mathrm{La}_{3}$ phase. The atomic radius of $\mathrm{Ni}$ is close to that of $\mathrm{Al}$. Thus, it is not unreasonable to suggest that the new cubic phase could be a bcc-(AlNi) ${ }_{11} \mathrm{La}_{3}$-like phase, which decomposes into some intermetallic compounds at higher temperatures. This cubic phase together with the fcc-Al phase are observed in all XRD patterns recorded after the first crystallization process at various pressures. These results indicate that the first crystallization process of the $\mathrm{Al}_{89} \mathrm{La}_{6} \mathrm{Ni}_{5}$ amorphous alloy might be governed by a eutectic reaction rather than a primary reaction, which was suggested in the literature. ${ }^{11} \mathrm{~A}$ similar two-phase precipitation process was also reported in the $\mathrm{Al}_{85} \mathrm{Y}_{11} \mathrm{Ni}_{4}$ amorphous alloy after the first crystallization process. ${ }^{14}$ More Bragg peaks appear in the XRD pattern recorded at $573 \mathrm{~K}$, indicating that the re-

TABLE I. Interplanar spacings of the metastable bcc-(AlNi) $)_{11} \mathrm{La}_{3}$-like phase with a lattice constant of $6.64 \AA$ and a space group of $\operatorname{Im} 3 \mathrm{~m}$ obtained from the in situ XRD pattern recorded at $1.5 \mathrm{GPa}$ and $483 \mathrm{~K}$ for the $\mathrm{Al}_{89} \mathrm{La}_{6} \mathrm{Ni}_{5}$ amorphous alloy.

\begin{tabular}{llc}
\hline \hline$h k l$ & $d_{\text {cal }}(\AA)$ & $d_{\text {exp }}(\AA)$ \\
\hline 110 & 4.695 & 4.69 \\
200 & $3.320^{\mathrm{a}}$ & \\
211 & 2.711 & 2.72 \\
220 & $2.348^{\mathrm{a}}$ & \\
400 & 1.660 & 1.66 \\
422 & 1.355 & 1.35 \\
\hline
\end{tabular}

${ }^{\mathrm{a}}$ Peaks are overlapped with those of $\mathrm{BN}$ or fcc $\mathrm{Al}$. Peaks are overlapped with those of BN or fcc Al. $\quad$ ergy. At ambient pressure, crystallization of an amorphous
Downloaded 12 Feb 2010 to 192.38.67.112. Redistribution subject to AlP license or copyright; see http://apl.aip.org/apl/copyright.jsp

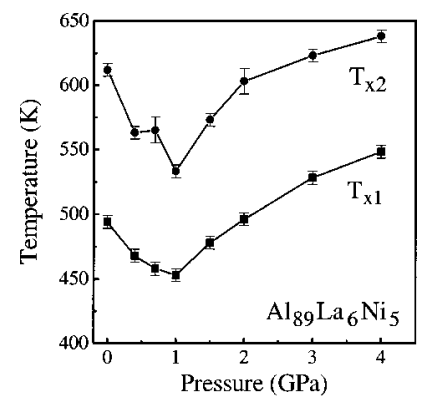

FIG. 2. Pressure dependences of the crystallization temperatures estimated from in situ XRD measurements for the $\mathrm{Al}_{89} \mathrm{La}_{6} \mathrm{Ni}_{5}$ metallic glass. The data at ambient pressure were estimated from the DSC measurement at a heating rate of $40 \mathrm{~K} / \mathrm{min}$.

sidual amorphous phase begins to crystallize and the bcc-(AlNi) ${ }_{11} \mathrm{La}_{3}$-like phase starts to decompose. At $673 \mathrm{~K}$, fcc- $\mathrm{Al}, \mathrm{Al}_{11} \mathrm{La}_{3}$, and $\mathrm{Al}_{3} \mathrm{Ni}$, together with a few tiny peaks, are detected while the bcc-(AlNi) ${ }_{11} \mathrm{La}_{3}$-like phase disappears. No room-temperature precipitation of fcc-Al crystals was observed recorded at pressures up to $4 \mathrm{GPa}$. Figure 2 displays $T_{x 1}$ and $T_{x 2}$ of the $\mathrm{Al}_{89} \mathrm{La}_{6} \mathrm{Ni}_{5}$ amorphous alloy as a function of the applied pressure, in which the values of $T_{x 1}$ and $T_{x 2}$ at ambient pressure were estimated from DSC at a heating rate of $40 \mathrm{~K} / \mathrm{min}$. The applied pressure strongly affects the crystallization temperatures of the amorphous $\mathrm{Al}_{89} \mathrm{La}_{6} \mathrm{Ni}_{5}$ alloy. However, pressure has no significant influence on the temperature difference between $T_{x 1}$ and $T_{x 2}$. Both temperatures first decrease with increasing pressure $(P<1 \mathrm{GPa})$, and then increase up to $4 \mathrm{GPa}$. The applied pressure has a similar effect on both crystallization processes in the pressure range studied.

Crystallization of an amorphous alloy is normally regarded as a process proceeding by nucleation and subsequent growth of crystals. During the initial stage of nucleation of crystals in the amorphous phase, the effect of pressure on the crystallization kinetics is associated with the atomic diffusion process and the volume change effect. The crystallization temperature of an amorphous alloy may be governed by the thermodynamic potential barrier of nucleation and diffusion activation energy. According to crystallization kinetics theory, the nucleation rate $I$ can be written as, $I=I_{0}$ $\times \exp \left(-\left(\Delta G^{*}+Q_{n}\right) / k T\right)$, where $I_{0}$ is a constant, $\Delta G^{*}$ is the free energy required to form a nucleus of the critical size, i.e., the thermodynamic potential barrier of nucleation, $Q_{n}$ is the activation energy for the transport of an atom across the interface of an embryo, and $k$ is the Boltzmann's constant. The sum $\Delta G^{*}+Q_{n}$ is called the nucleation work. For homogeneous nucleation, $\Delta G^{*}$ at a given pressure $P$ can be expressed by

$\Delta G^{*}(T, P)=\frac{16 \pi \sigma^{3}\left(V_{m}^{c}\right)^{2}}{3\left[P\left(V_{m}^{a}-V_{m}^{c}\right)-\left(\Delta G^{a \rightarrow c}(T, P)+E\right)\right]^{2}}$.

where $V_{m}^{c}$ and $V_{m}^{a}$ are the molar volumes of the crystalline and amorphous phases, respectively, $\Delta G^{a \rightarrow c}(T, P)$ is the molar free energy change for the transformation from the amorphous to the crystalline phase, $E$ is the elastic energy induced by the volume change during the amorphous-tocrystalline phase transformation, and $\sigma$ is the interfacial en- 


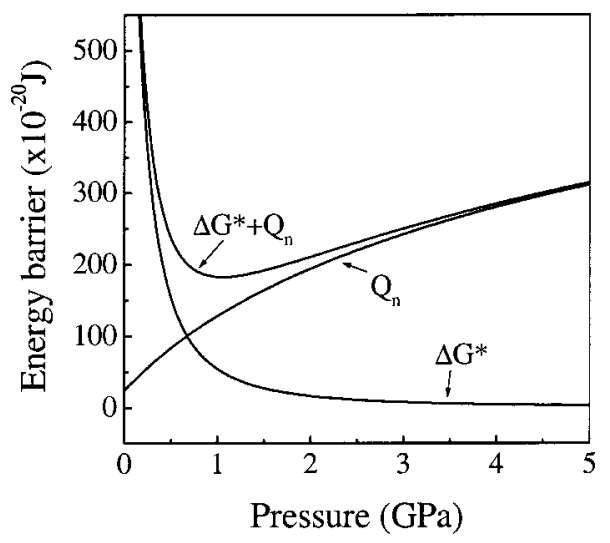

FIG. 3. Schematic illustration of the pressure dependences of $\Delta G^{*}, Q_{n}$, and $\Delta G^{*}+Q_{n}$.

alloy involves the formation of crystalline phase(s) with higher density, ${ }^{15}$ i.e., $V_{m}^{c}<V_{m}^{a}$. For the $\mathrm{Al}_{89} \mathrm{La}_{6} \mathrm{Ni}_{5}$ amorphous alloy, they are $V_{m}^{c}=10.0 \mathrm{~cm}^{3} / \mathrm{mol}$ for the fcc-Al and $V_{m}^{a}=11.6 \mathrm{~cm}^{3} / \mathrm{mol}$ for the amorphous alloy. ${ }^{8}$ Both $V_{m}^{c}$ and $V_{m}^{a}$ have a similar pressure dependence. ${ }^{16}$ Assuming a negligible pressure dependence of $\Delta G^{a \rightarrow c}(T, P), E$, and $\sigma$, it follows that $\Delta G^{*}$ decreases with increasing pressure, thus promoting crystallization. On the other hand, applied pressure, in general, reduces the atomic mobility, suggesting that $Q_{n}$ increases with increasing pressure. When the amorphous alloy crystallizes in a eutectic mode, atoms in the amorphous alloy need to rearrange. Thus, the enhancement of $Q_{n}$ with pressure retards the crystallization process. Therefore, the role of pressure on the nucleation during crystallization process of the $\mathrm{Al}_{89} \mathrm{La}_{6} \mathrm{Ni}_{5}$ amorphous alloy might be governed by the two factors. The competing processes can be applied to qualitatively explain the experimental results obtained here for the $\mathrm{Al}_{89} \mathrm{La}_{6} \mathrm{Ni}_{5}$ amorphous alloy. Figure 3 schematically illustrates the variation of $\Delta G^{*}, Q_{n}$, and $\Delta G^{*}+Q_{n}$ with pressure, in which $Q_{n}(\mathrm{P}=0)=1.53 \mathrm{eV}$ (Ref. 17) and it is assumed to gradually increase with pressure. $\Delta G^{*}$ is calculated from Eq. (1) for the $\mathrm{Al}_{89} \mathrm{La}_{6} \mathrm{Ni}_{5}$ amorphous alloy using $\sigma=0.108 \mathrm{~J} / \mathrm{m}^{2}$ and $\Delta G^{a \rightarrow c}(T, P)=144 \mathrm{~J} / \mathrm{cm}^{3} .{ }^{8}$ $\mathrm{E} \approx \mathrm{G} \varepsilon^{2} V_{m}^{c} / 2$, where $\mathrm{G}$ is Young's modulus of $75 \mathrm{GPa}$ for the amorphous alloy ${ }^{18}$ and $\varepsilon=\left(V_{m}^{a}-V_{m}^{c}\right) /\left(3 V_{m}^{c}\right)$. The elastic energy during the amorphous-to-crystalline phase transformation could be smaller than the estimated value due to structural relaxation, which does not alter the following qualitative explanation. At low pressures $(0-1 \mathrm{GPa}), \Delta G^{*}$ is much larger than $Q_{n}$ and the dominant factor. Thus, the nucleation work decreases with pressure. This results in an enhancement of $I$ and a reduction of the crystallization temperature with pressure, as observed in our study. With increasing pressure, $\Delta G^{*}$ rapidly decreases while $Q_{n}$ increases so that atomic diffusion becomes dominant factor in the nucleation process. Hence, the nucleation work increases with pressure. Consequently, nucleation rate decreases with pressure and an enhancement of crystallization temperature is expected, as observed for the $\mathrm{Al}_{89} \mathrm{La}_{6} \mathrm{Ni}_{5}$ amorphous alloy in the pressure range of $1-4 \mathrm{GPa}$.
In summary, the crystallization of the $\mathrm{Al}_{89} \mathrm{La}_{6} \mathrm{Ni}_{5}$ amorphous alloy has been investigated by in situ high-pressure and high-temperature $\mathrm{x}$-ray powder diffraction measurements using synchrotron radiation in the pressure range of 0-4 GPa. The first crystallization process of the $\mathrm{Al}_{89} \mathrm{La}_{6} \mathrm{Ni}_{5}$ amorphous alloy, corresponding to simultaneous precipitation of fcc-Al crystals and the metastable bcc-(AlNi) $)_{11} \mathrm{La}_{3}$-like phase, may be a eutectic reaction. The second one corresponds to the transformation of the residual amorphous alloy into fcc- $\mathrm{Al}, \mathrm{Al}_{11} \mathrm{La}_{3}, \mathrm{Al}_{3} \mathrm{Ni}$, and as yet unidentified phase(s). $T_{x 1}$ and $T_{x 2}$ first decrease with pressure in the pressure range of $0-1 \mathrm{GPa}$ and then increase with pressure up to $4 \mathrm{GPa}$. The results were discussed with reference to competing processes between the thermodynamic potential barrier and the diffusion activation energy under pressure.

${ }^{1}$ A. Inoue, K. Ohtera, A. P. Tsai, and T. Masumoto, Jpn. J. Appl. Phys., Part 2 27, L280 (1998); 27, L479 (1988).

${ }^{2}$ Y. He, S. J. Poon, and G. J. Shiflet, Science 241, 1640 (1988); G. J. Shiflet, Y. He, and S. J. Poon, J. Appl. Phys. 64, 6863 (1988).

${ }^{3}$ H. Chen, Y. He, G. J. Shiflet, and S. J. Poon, Scr. Metall. Mater. 25, 1421 (1991)

${ }^{4}$ Y. H. Kim, A. Inoue, and T. Masumoto, Mater. Trans., JIM 32, 331 (1991); A. Inoue and H. Kimura, Mater. Sci. Forum 235-238, 873 (1997); A. Inoue, Prog. Mater. Sci. 43, 365 (1998).

${ }^{5}$ T. Gloriant and A. L. Greer, Nanostruct. Mater. 10, 389 (1998); Z. C. Zhong and A. L. Greer, Int. J. Non-Equilib. Process. 11, 35 (1998).

${ }^{6}$ F. Zhou, X. H. Zhang, and K. Lu, J. Mater. Res. 13, 784 (1998).

${ }^{7}$ W. K. Wang, H. Iwasaki, C. Suryanarayana, and T. Masumoto, J. Mater. Sci. 18, 3765 (1983).

${ }^{8}$ F. Ye and K. Lu, Acta Mater. 47, 2449 (1999); Phys. Rev. B 60, 7018 (1999); Acta Mater. 46, 5965 (1998).

${ }^{9}$ J. Z. Jiang, J. S. Olsen, L. Gerward, S. Abdali, J. Eckert, N. Schlorke-de Boer, L. Schultz, and P. X. Shi, J. Appl. Phys. 87, 2664 (2000); J. Z. Jiang, T. J. Zhou, H. Rasmussen, U. Kuhn, J. Eckert, and C. Lathe, Appl. Phys. Lett. 77, 3553 (2000)

${ }^{10}$ H. Chen, Y. He, G. J. Shiflet, and S. J. Poon, Nature (London) 367, 541 (1994); A. A. Csontos and G. J. Shiflet, Nanostruct. Mater. 9, 281 (1997).

${ }^{11}$ F. Ye and K. Lu, J. Non-Cryst. Solids 262, 228 (2000).

${ }^{12}$ M. Mezouar, T. Le Bihan, H. Libotte, Y. Le Godec, and D. Häusermann, J. Synchrotron Radiat. 6, 1115 (1999).

${ }^{13}$ Y. Le Godec, D. M. Garcia, M. Mezouar, G. Syfosse, J. P. Itie, and J.-M. Besson (unpublished).

${ }^{14}$ M. Gogebakan, P. J. Warren, and B. Cantor, Mater. Sci. Eng., A 226-228, 168 (1997).

${ }^{15}$ H. S. Chen, J. Appl. Phys. 49, 3289 (1978).

${ }^{16}$ In a dense random packed structure, the position of the main broad $\mathrm{x}$-ray diffraction maximum (in $\AA^{-1}$ ) is proportional to $1 / d$, where $d$ is the nearest neighbor distance in the amorphous alloy deduced from the position of the first peak in the radial distribution function curve (in $\AA$ ). If one assumes that the density of the amorphous alloy is proportional to $1 / d^{3}$, one can estimate the ratio of the volume at a given pressure to that at zero pressure, $V(p) / V(0)$, of the amorphous alloy. We plotted the $V(p) / V(0)$ vs pressure for both the amorphous alloy at temperatures just below $T_{x 1}$ and the fcc-Al phase at temperatures just above $T_{x 1}$ and revealed that both phases have an almost identical pressure dependence.

${ }^{17}$ M. B. Bewersdorff, J. Mater. Sci. Lett. 10, 1225 (1991).

${ }^{18}$ A. Inoue, H. M. Kimura, K. Sasamori, and T. Masumoto, Sci. Rep. Res. Inst. Tohoku Univ. A 42, 165 (1996). 